\title{
Análisis multidimensional de los portales de datos abiertos autonómicos españoles
}

\author{
Ricardo Curto-Rodríguez
}

Universidad de Oviedo, Facultad de Comercio, Turismo y Ciencias Sociales "Jovellanos", Gijón. Correo-e: curtoricardo@uniovi.es I ID ORCID: https://orcid.org/0000-0002-6628-5278

Resumen: Iniciativas de Gobierno Abierto y de reutilización de la información del sector público están implementadas en la mayor parte de los países. Los portales de open data, repositorios digitales que centralizan la información en manos de las administraciones públicas bajo las premisas de formatos abiertos y licencias libres, son el destino de estos datos. En España el desarrollo de los portales de datos abiertos autonómicos es innegable, pasando de 11 portales operativos y una oferta aproximada de 5.000 datasets en el año 2013, a 17 repositorios y más de 14.000 conjuntos de datos en 2019. Este estudio se ocupa de valorar estos portales de datos abiertos autonómicos considerando cuatro dimensiones complementarias: número de datasets, aplicaciones elaboradas en base a esos conjuntos de datos, opciones de interacción disponibles y funcionalidad observada en el portal, encontrando importantes diferencias intracomunitarias.

Palabras clave: datos abiertos; reutilización; colaboración; Comunidades Autónomas; España

\section{Multidimensional analysis of Spanish Autonomic Open Data Portals.}

Abstract: Open Government initiatives and reuse of public sector information are implemented in most countries. The destination of this data are open data portals, digital repositories that centralize information of public administrations under the premises of open formats and free licenses. In Spain, the development of regional governments open data portals is undisputed, going from 11 open portals with an approximate offer of 5.000 datasets in 2013, to 17 repositories and more than 14.000 datasets in 2019. This study evaluates these regional government open data portals, considering four complementary dimensions: number of datasets, applications developed, based on these datasets, available interaction options and observed functionality in the portal, finding important intra-community differences.

Keywords: open data; data reuse; collaboration; Regional Governments; Spain

Cómo citar este artículo/Citation: Curto-Rodríguez, R. (2021). Análisis multidimensional de los portales de datos abiertos autonómicos españoles. Revista Española de Documentación Científica, 44 (1), e284. https://doi.org/ 10.3989/redc.2021.1.1745

Copyright: (c) 2021 CSIC. Este es un artículo de acceso abierto bajo los términos de la licencia de uso y distribución Creative Commons Reconocimiento 4.0 Internacional (CC BY 4.0). 


\section{INTRODUCCIÓN}

Los datos son la gran revolución de nuestro tiempo. Big data, Data mining, Open data, Research data, Linked data, Open linked data, Data science, Data literacy, Social data, Data infrastructure, Data privacy son términos que con frecuencia se utilizan en los medios de comunicación (Hernández-Pérez, 2016; Aleixandre-Benavent y otros, 2019), teniendo todos ellos un punto en común, el empleo de la información.

La era de los datos ya está aquí, brindando oportunidades para desarrollar negocios y conocimiento (Peset y otros, 2017; Manfredi, 2017) siendo "the new oil for the digital age" (Kroes, 2012) y creando una nueva fiebre del oro (Hernández-Pérez, 2016). No en vano informes como el encargado por la Comisión Europea a Vickery (2011) valoran una creación de riqueza para la zona euro de 40.000 millones al año (140.000 millones/año si incorporamos el impacto indirecto), generando en España entre 600 y los 750 millones (ONTSI, 2016).

Estos datos tienen muchas utilidades: incremento de la interoperabilidad de las distintas administraciones, generación de valor añadido mediante la creación de nuevos servicios digitales, aumento de la transparencia por la publicación de información de tipo económico y legislativo, mejora de la participación ciudadana al posibilitar la formación de opiniones y la colaboración, o una mayor gobernanza abierta y de servicio (Curto-Rodríguez, 2017a). Todo ello ha sido posible porque ha cambiado la forma en que el sector público gestiona la información al pasar de acumularla para su uso interno, a ponerla a disposición de los ciudadanos (Mendo y otros, 2013), desarrollando un nuevo ecosistema asociado a los datos abiertos (figura 1).

Se trata, por tanto, de ir un paso más allá de la administración electrónica para que se pueda reutilizar la información pública y explorar las posibilidades de los big data o del data mining (Boix, 2015) mediante diversas técnicas de aprovechamiento de la información (Ferrer-Sapena y Sánchez-Pérez, 2013). El requisito para fomentar el empoderamiento y la participación ciudadana es que la información en línea cumpla unos criterios, elaborados en base a los ocho principios sintetizados por la Sunlight Foundation (2007), de calidad, usabilidad y accesibilidad, de forma que el ciudadano logre encontrarla, revisarla, analizarla y finalmente reutilizarla (Martínez Moya, 2015; Cerrillo-Martínez, 2018).

No cabe duda, el uso de los datos se intensifica cada año (Ramos-Simón, 2017). Por ello, y para centralizar la información, se han creado unos portales de datos abiertos configurando una tendencia de apertura de repositorios que, a nivel español,

Figura 1: tendencias presentes y futuras en el mundo de los datos

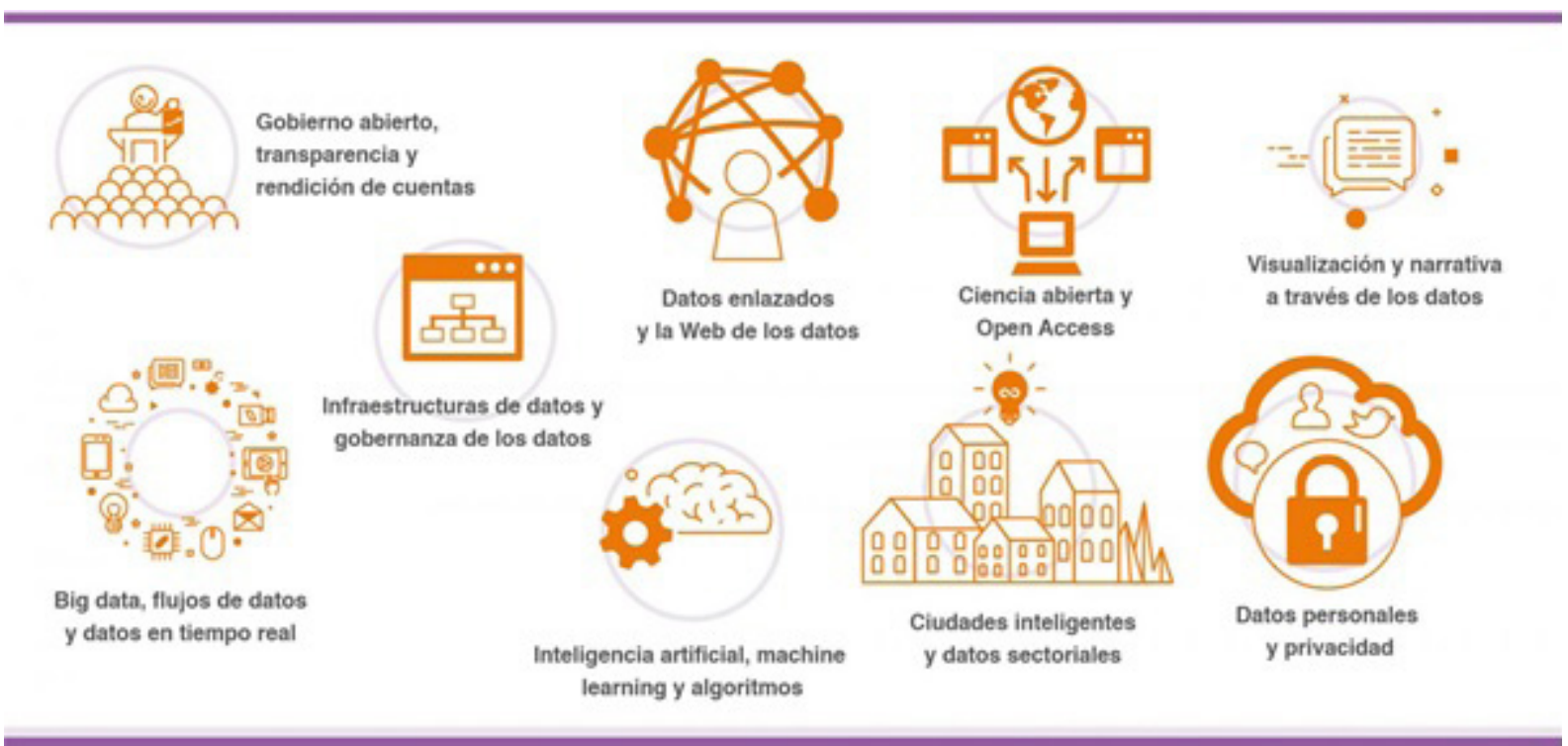

Fuente: https://datos.gob.es/es/noticia/el-futuro-de-los-datos-abiertos-y-sus-multiples-caras 
se traduce en casi 300 portales operativos, según indica datos.gob.es (2019), y de 2.600 en clave mundial, según identifica el estudio Open data inception (2019).

Lejos ha quedado ya 2013 y la eclosión de los portales en España, cuando se escuchaban expresiones como la de David Cabo (2013): "Portales opendata como aeropuertos sin aviones", indicando que los políticos, al igual que se apresuraron a levantar grandes obras de infraestructuras aeroportuarias hoy abandonadas, inauguraron portales de datos abiertos para aparentar transparencia, o la de Gestores Públicos (2013): "Portales Open Data en las Comunidades Autónomas : Mucha propaganda pero todavía poca información útil". Nos parece interesante, por tanto, evaluar qué ha pasado en estos seis últimos años y valorar la situación actual.

Para responder a estos interrogantes analizaremos de forma multidimensional los portales de datos abiertos autonómicos españoles, observando la divulgación de conjuntos de datos reutilizables de forma que puedan ser libremente procesados por los usuarios como materia prima para la generación de servicios digitales o aplicaciones informáticas, y fomentando los procesos de colaboración ciudadana.

El trabajo se desarrolla siguiendo la siguiente estructura. En la segunda sección se describe brevemente el marco conceptual en el que se circunscribe la investigación, ocupándose la tercera parte del indicador empleado para valorar los portales y del proceso de recogida de resultados. El cuarto apartado analiza los principales resultados, que son seguidos por las principales conclusiones obtenidas y por las referencias bibliográficas empleadas en el estudio.

\section{MARCO TEÓRICO Y ESTUDIOS SOBRE PORTALES DE DATOS ABIERTOS}

Las tecnologías de la información y de la comunicación están transformando las administraciones públicas (Criado Grande, 2016). Los gobiernos de todo el mundo intentan crear valor utilizando estrategias emergentes, disruptivas e inteligentes (Criado y Gil-García, 2019) que tienen el potencial de fomentar la cocreación de servicios públicos gracias a su naturaleza colaborativa, social y horizontal (Ruvalcaba-Gómez y otros, 2019). Este desarrollo tecnológico permite cada vez más una ciencia basada en los datos y facilita el impulso político a la open science (Gómez y otros, 2016) en un entorno en el que las administraciones públicas son unas de las principales creadoras de datos (Cerrillo-Martínez, 2018).
Pero este impulso a los datos ha llegado también desde otras dimensiones como la política, siendo inexcusable no mencionar los memorándums del presidente norteamericano (Obama 2009a; 2009b), quien puso en práctica la formulación original de gobierno abierto u Open Goverment, atribuida a Parks (1957), bajo los pilares de la transparencia, la colaboración y la participación.

No podemos olvidar tampoco la vertiente legislativa, ya que hemos pasado de 13 países con normas sobre el acceso a la información en los años 90 a cerca de 100 en la actualidad, según señala la UNESCO. En España, la Ley 19/2013 de transparencia, acceso a la información pública y buen gobierno (LTAIPyBG) se fundamenta en la filosofía del open goverment y los open data (Beltrán Orenes y Martínez Pastor, 2016), y ha conseguido acrecentar el interés sobre estas materias (Sierra Rodríguez, 2018a), convirtiéndolas poco a poco en una realidad (Sierra Rodríguez, 2018b).

Hoy en día la LTAIPYBG ha sido desarrollada y complementada por todas las Comunidades Autónomas (CC.AA.) españolas mediante leyes específicas y por otra serie de iniciativas como planes, leyes, acuerdos, agendas y estrategias de gobierno abierto consultables en Alcaide Muñoz y otros, (2019). Recomendamos examinar los principios de los datos abiertos en la legislación española (Cerillo-Martínez, 2014), o el compendio de normativas asociadas con la reutilización de la información del sector público realizado por González Limón y Rodríguez-Ramos (2019), para un mayor conocimiento de las leyes que regulan los datos abiertos en España.

Todo lo comentado ha generado una tendencia internacional hacia la apertura de datos (Clabo y Ramos Vielba, 2005) y un interés en el estudio de estos datos abiertos u open data, así como de los repositorios que los albergan: los portales de datos abiertos u open data portals. Mostramos a continuación varios de ellos comenzando por una de las investigaciones pioneras, la de Ramos Simón y otros, (2012) que, entre abril y octubre de 2012, analizan diez portales nacionales europeos, siendo el del Reino Unido el que presenta una mejor propuesta y un uso eficiente de los datos públicos.

De gran interés son una serie de investigaciones realizadas fuera de España como la de Thorsby y otros, (2016) que analizan las características y el contenido de los portales de datos abiertos en las ciudades estadounidenses mediante la creación de cinco escalas de valoración, afirmando que los portales se encuentran en una etapa muy temprana de desarrollo y que necesitan una gran cantidad de trabajo para mejorar la ayuda al usuario, las fun- 
ciones de análisis, y la incorporación de gráficos, siendo el tamaño poblacional (medido por el nivel de recursos disponibles locales) un factor crítico que está asociado a las tres variables dependientes utilizadas. También existen otros trabajos que pretenden rellenar un knowlegde gap en cuanto a la capacidad de los portales de open data para el estímulo de la coproducción ciudadana como Chatfield y Reddick (2017), que analizan los veinte portales locales de datos abiertos de las ciudades más grandes de Australia encontrando variaciones relevantes en cuanto a las posibilidades de generar servicios abiertos o Sandoval-Almazán y Gil García (2012), que muestran, en clave local mejicana, que los portales de Internet están realizando un progreso muy lento de evolución hacia una mayor interacción, participación y colaboración, calificándolo como retórico en algunos casos.

Otros estudios que se ocupan de los datos abiertos a nivel internacional son los resumidos por $\mathrm{Vi-}$ cente-Paños y Jordán-Alfonso (2017) permitiendo analizar aspectos claves de las distintas iniciativas y comparativas entre países como: Open Data Index (https://index.okfn.org/methodology/), Open Data Barometer (https://opendatabarometer.org) u Open Data Monitor (https://opendatamonitor. eu). Destacamos asimismo el informe que publica anualmente (un informe por año para el periodo 2015-2019) el portal de datos europeo sobre el nivel de madurez de los datos abiertos en los estados miembros de la UE, mas Islandia, Liechtenstein, Noruega y Suiza, en el que España ocupa la segunda posición (https://www.europeandataportal.eu/ es/dashboard\#2019) denominado Open Data Maturity. Por su parte, Arquero Avilés y Marco Cuenca (2014) se ocupan de realizar un análisis del Portal de datos abiertos de la Unión Europea centrándose en su contenido, los estándares en los que se basa, así como los principales conjuntos de datos, formatos y aplicaciones que ofrece.

No obstante, preferimos centrarnos en las investigaciones relacionadas con los datos abiertos en España, siendo destacable la existencia de un número reducido de trabajos que son resumidos a continuación en orden cronológico.

Uno de los primeros estudios es el de Curto-Rodríguez (2015) que repasa diversos impulsos del gobierno abierto y de los datos abiertos tanto políticos como de diversos organismos, mostrando un mapa de las principales iniciativas a nivel mundial y español y realizando un análisis comparativo de los portales de las CC.AA. para los meses de noviembre y diciembre de 2013 (y revisión de enero de 2014). Tras valorar la cantidad de información y calidad reutilizadora existente, según el esquema 5 star open data, (Berners-Lee, 2010), señala como líder destacado al País Vasco en cuanto al suministro de información asociada a la transparencia activa. El mismo autor califica como prácticamente intranscendente el efecto de la entrada en vigor de la LTAIPYBG en cuanto a la divulgación en los portales de datos abiertos (Curto-Rodríguez, 2017a) estudiando en profundidad los cambios ocurridos en el periodo 2013-2015 (Curto-Rodríguez, 2017b).

Por su parte Martínez-Méndez y otros, (2014) muestran una visión general de los open data en las administraciones públicas españolas en marzo de 2014, analizando datasets y apps existentes a partir del portal datos.gob.es. El estudio identifica un elevado volumen de categorías temáticas que recogen datos de muy diversos tipos y un reducido nivel de desarrollo de aplicaciones.

Con el título "Datos abiertos en las Comunidades Autónomas y sus mayores ayuntamientos 2014", la Fundación Orange y Arvo consultores (2014) visita, para el periodo enero-marzo de 2014, los portales de open data españoles elaborando un completo informe. El estudio analiza las 11 CC.AA. con portal aperturado (Andalucía, Aragón, Baleares, Castilla-La Mancha, Castilla y León, Cataluña, Extremadura, Galicia, La Rioja, Comunidad Foral de Navarra y País Vasco), siguiendo una metodología basada en tres ejes: los principios de gobierno abierto (transparencia, participación y colaboración); la dimensión técnica, según el esquema 5 star open data; y la dimensión valor, bajo un prisma económico, social y político.

Vicente-Paños y Jordán-Alfonso (2017) realizan, en el año 2016, un muestreo estratificado proporcional de los 7.098 conjuntos de datos existentes en las $15 \mathrm{CC}$. AA con portal operativo, analizando un total 2.165 datasets y empleando la métrica MELODA (Abella y otros, 2014), para apuntar una excelente facilidad de reúso, ya que el $95 \%$ de los datasets presentaban una reutilización avanzada o avanzada con alguna característica mejorable.

Los mismos autores de MELODA (Abella y otros, 2018), tras indicar que Janowicz y otros (2014) afirman que el esquema 5 star open data constituye una adecuada referencia para valorar el potencial de uso de los datos de las instituciones, deciden emplear este indicador para analizar el portal de open data de la ciudad de Barcelona, que en 2017 tenía 425 datos y 3.002 distribuciones. El estudio indica que casi la totalidad de datasets (un 98\%) ofrecen formatos dos estrellas (que en general se corresponden a un fichero XLS), o llegan a alcanzar las tres estrellas $(85,2 \%$ ) (que de forma habitual se corresponden con un fichero CSV). Otros estudios a mencionar serían Abella y otros, (2017), que 
realizan un análisis sobre la reutilización de datos abiertos en España revisando 103 portales operativos, los conjuntos de datos alojados y su uso, o Abella y otros, (2019), que analizan los cambios y avances producidos en los dos últimos años, identificando directrices y emitiendo diversas recomendaciones.

También existe una importante línea de investigación de la información por medios electrónicos relacionada con la transparencia activa. García-García y Curto-Rodríguez (2018a y 2018b) visitan los portales de datos abiertos, los portales de transparencia y las webs autonómicas en 2013 en busca de los determinantes de una mayor divulgación. El análisis continúa con una exhaustiva revisión en 2017 de los portales de open data (García-García y Curto-Rodríguez, 2019), quienes incorporan posteriormente los portales de transparencia y la web institucional (García-García y Curto-Rodríguez, 2018b). Estos trabajos identifican, de acuerdo con las teorías de la agencia y neoinstitucional, comportamientos de transparencia sincera y de transparencia aparente.

Por último, González-Limón y Rodríguez-Ramos (2019) analizan el cumplimiento por parte de las entidades locales de los 20 datos mínimos acordados por la Federación Española de Municipios y Provincias (FEMP), 2017), constatando que tan solo trece ayuntamientos son publicadores en España, de los cuales, solo cumplen con el $100 \%$ de los datasets exigidos Madrid y Zaragoza (si bien el $75 \%-90 \%$ es atendido por Gijón, Barcelona, Vitoria-Gasteiz y Bilbao). Recientemente (FEMP, 2019) ha identificado otros 20 conjuntos de datos dejando en un total de 40 los datos mínimos a publicar e indicando cómo deberían ser publicados.

Tras la revisión de la principal literatura relacionada con los portales de datos abiertos en nuestro país, procede contextualizar nuestro estudio. Lo hemos enfocado en las CC.AA. españolas ya que España es un país fuertemente descentralizado, prácticamente cuasifederal (Bastida y otros, 2019), donde las autonomías constituyen el segundo subsector de mayor importancia dentro del sector institucional de las administraciones públicas y con un peso muy cercano al primero, los fondos de la Seguridad Social (Melián González y otros, 2018).

Por ello, las CA.AA. cuentan con un tamaño suficiente que les permite disponer de recursos humanos y financieros para abordar proyectos innovadores como los portales de open data. No obstante, el hecho de que las autonomías sean muy distintas entre sí en población, superficie, nivel de renta, identidad histórica, lingüística y cultural (Subirats, 1995) nos invita a pensar que existirán importan- tes diferencias en sus iniciativas de datos abiertos, cuestión que valoraremos en el siguiente apartado.

\section{METODOLOGÍA, INDICADOR EMPLEADO Y RESULTADOS POR APARTADO}

El desempeño de los portales de datos abiertos es un concepto poliédrico. Por ello, hemos creado un indicador que considere diversas facetas: el número de datasets, los servicios digitales desarrollados a partir de dichos conjuntos de datos, las opciones de interacción disponibles en los repositorios, así como diversas funcionalidades que faciliten su utilización.

El trabajo de campo fue realizado a finales de 2019 (diciembre para la cuantificación de los datasets). La Tabla I ofrece los conjuntos de datos de forma comparativa a los existentes en 2013, 2015 y 2017.

A continuación, se recogió el número de aplicaciones disponibles en cada uno de los portales de datos abiertos autonómicos (Tabla II) mostrando, por un lado, el total de aplicaciones ofertadas (muchas de ellas sin relación con la información suministrada por el portal de la CC.AA.) y, por otro, las desarrolladas a partir de los datasets allí alojados, que serán las contempladas por nuestro estudio.

En tercer lugar, nos ocupamos de indagar sobre las posibilidades de interacción habilitadas en los repositorios autonómicos que van a permitir mantener una comunicación bidireccional con los responsables de los portales de open data, como la solicitud de información, las redes sociales o el aviso de novedades (tabla III). El cuarto aspecto a estudiar se muestra en la Tabla IV recogiendo una serie de prestaciones o funcionalidades que permiten un mejor aprovechamiento del portal de datos abiertos.

\section{ANÁlISIS DE RESULTADOS Y CONTEX- TUALIZACIÓN, VALORACIÓN GLOBAL DE LOS REPOSITORIOS Y CLASIFICACIÓN AU- TONÓMICA}

\subsection{Datasets}

Como ha mostrado la Tabla I, hemos podido apreciar que las CC.AA. españolas se han sumado a la iniciativa de los datos abiertos disponiendo todas de portal de open data operativo (si bien Cantabria con versión Beta). Estas CC.AA. son las mayores suministradoras de datos abiertos en nuestro país al ofrecer en 2019 más de 14.000 conjuntos de datos (gracias a un incremento sostenido de 3.000 datasets bianuales que le ha llevado casi a triplicar los 5.000 datasets existentes en 2013). Además, la sindicación de las CC.AA. supera a la administración general del Estado, a las administraciones lo- 
Tabla I: Portales de datos abiertos autonómicos y conjuntos de datos en diciembre de 2013, 2015, 2017 y 2019

\begin{tabular}{|c|c|c|c|c|c|}
\hline Comunidad Autónoma & Nombre del Portal & dic-13 & dic-15 & dic-17 & dic-19 \\
\hline Andalucía & Datos Abiertos & 70 & 178 & 464 & 555 \\
\hline Aragón & Aragón Open Data & 440 & 2519 & 2794 & 2824 \\
\hline Canarias & Datos Abiertos Canarias & Sin Portal & 45 & 43 & 186 \\
\hline Cantabria & Icane - datos (versión beta) & Sin portal & Sin portal & Sin portal & 356 \\
\hline Castilla y León & Datos Abiertos de Castilla y León & 124 & 176 & 334 & 488 \\
\hline Castilla-La Mancha & Datos abiertos de Castilla- La Mancha & 42 & 59 & 207 & 271 \\
\hline Cataluña & Datos Abiertos GenCat & 1460 & 1468 & 571 & 578 \\
\hline Comunidad de Madrid & Comunidad de Madrid datos abiertos & Sin Portal & Sin Portal & Sin Portal & 178 \\
\hline Com. Foral de Navarra & Open Data & 135 & 190 & 1186 & 1378 \\
\hline Comunidad Valenciana & Dades obertes gva & Sin Portal & 279 & 338 & 492 \\
\hline Extremadura & Gobierno Abierto - Catálogo de datos & 26 & 25 & 25 & 25 \\
\hline Galicia & abert@s & 292 & 324 & 349 & 366 \\
\hline Islas Baleares & Dades obertes caib & 42 & 42 & 42 & 182 \\
\hline La Rioja & Datos Abiertos Rioja & 64 & 86 & 265 & 318 \\
\hline País Vasco & Opendataeuskadi & 2280 & 2242 & 4123 & 4970 \\
\hline Principado de Asturias & Open Data del Principado de Asturias & Sin Portal & 419 & 42 & 72 \\
\hline Región de Murcia & Datos abiertos Región de Murcia & Sin Portal & Sin Portal & 271 & 765 \\
\hline \multicolumn{2}{|l|}{ TOTALES } & 4975 & 8052 & 11054 & 14004 \\
\hline
\end{tabular}

Tabla II: Aplicaciones albergadas en los portales de open data y aplicaciones desarrolladas exclusivamente a partir de los conjuntos de datos autonómicos

\begin{tabular}{|c|c|c|c|}
\hline $\begin{array}{l}\text { Comunidad } \\
\text { Autónoma }\end{array}$ & Localización de las aplicaciones & $\begin{array}{l}\text { Total } \\
\text { Apps }\end{array}$ & $\begin{array}{c}\text { Apps } \\
\text { Autonómicas }\end{array}$ \\
\hline Andalucía & Pestaña "aplicaciones" en página principal & 89 & 24 \\
\hline Aragón & Enlace "aplicaciones" en página principal & 15 & 13 \\
\hline Canarias & Pie de página "aplicaciones" en página principal & 0 & 0 \\
\hline Cantabria & No existe apartado & 0 & 0 \\
\hline Castilla y León & Pestaña "aplicaciones" en página principal & 36 & 19 \\
\hline Castilla-La Mancha & No existe apartado & 0 & 0 \\
\hline Cataluña & Enlace" ¿Qué se está haciendo con los datos abiertos?" en página principal & 16 & 16 \\
\hline Comunidad de Madrid & Enlace "visores de datos abiertos" en página principal & 4 & 4 \\
\hline Com. Foral de Navarra & Enlace "aplicaciones datos abiertos" en página principal & 17 & 5 \\
\hline Comunidad Valenciana & No existe apartado & 0 & 0 \\
\hline Extremadura & No existe apartado & 0 & 0 \\
\hline Galicia & Menú ideas (submenú experiencias) en la página principal & 9 & 3 \\
\hline Islas Baleares & No existe apartado & 0 & 0 \\
\hline La Rioja & Apartado "aplicaciones" en la página principal & 4 & 4 \\
\hline País Vasco & Pestaña "cómo reutilizar (submenú ideas y ejemplos)" en página principal & 9 & 3 \\
\hline Principado de Asturias & No existe apartado & 0 & 0 \\
\hline Región de Murcia & Pestaña "aplicaciones" en página principal & 1 & 1 \\
\hline \multicolumn{2}{|l|}{ TOTALES } & 200 & 92 \\
\hline
\end{tabular}


Tabla III: Posibilidades de interacción presentes en los portales de datos abiertos autonómicos españoles

\begin{tabular}{|c|c|c|c|c|c|c|c|c|c|c|c|c|c|c|c|c|c|}
\hline INTERACCIÓN & AND & ARA & AST & BAL & CAT & CNR & CNT & CTL & CTM & EXT & GAL & MAD & MUR & NAV & RIO & VAL & VAS \\
\hline \multicolumn{18}{|l|}{ Solicitud de información } \\
\hline $\begin{array}{l}\text { Se facilita forma de } \\
\text { contacto }\end{array}$ & $x$ & $x$ & $x$ & $x$ & $\mathrm{x}$ & $\mathrm{x}$ & & $\mathrm{x}$ & $x$ & & $\mathrm{x}$ & $x$ & $\mathrm{x}$ & $\mathrm{x}$ & $X$ & $\mathrm{x}$ & $\mathrm{x}$ \\
\hline $\begin{array}{l}\text { Blog o Herramienta de } \\
\text { votación de propuestas }\end{array}$ & & $x$ & & & & & & $x$ & & & & & & & & & \\
\hline \multicolumn{18}{|l|}{ Redes sociales específicas } \\
\hline Twitter & $x$ & & & & $x$ & & & $x$ & & & & & & & & & $x$ \\
\hline $\begin{array}{l}\text { Sindicación de contenidos } \\
\text { (RSS) o Subscripción de } \\
\text { novedades }\end{array}$ & & & & & $x$ & & $x$ & $x$ & & $x$ & $x$ & $\mathrm{x}$ & & $x$ & & & \\
\hline
\end{tabular}

NOTA: AND - Andalucía, ARA - Aragón, AST - Principado de Asturias, BAL - Islas Baleares, CAT - Cataluña, CNR - Canarias, CNT - Cantabria, CTL - Castilla y León, CTM - Castilla-La Mancha, EXT - Extremadura, GAL - Galicia, MAD - Comunidad de Madrid, MUR - Región de Murcia, NAV - Comunidad Foral de Navarra, RIO - La Rioja, VAL - Comunidad Valenciana, VAS - País Vasco.

Tabla IV: Utilidades o funcionalidades habilitadas en los portales de datos abiertos autonómicos españoles

\begin{tabular}{|l|c|c|c|c|c|c|c|c|c|c|c|c|c|c|c|c|c|}
\hline \multicolumn{1}{|c}{ FUNCIONALIDADES } & AND & ARA & AST & BAL & CAT & CNR & CNT & CTL & CTM & EXT & GAL & MAD & MUR & NAV & RIO & VAL & VAS \\
\hline Diseño moderno y funcional & $\mathrm{x}$ & $\mathrm{x}$ & & & $\mathrm{x}$ & $\mathrm{x}$ & $\mathrm{x}$ & $\mathrm{x}$ & $\mathrm{x}$ & $\mathrm{x}$ & $\mathrm{x}$ & $\mathrm{x}$ & $\mathrm{x}$ & $\mathrm{x}$ & $\mathrm{x}$ & $\mathrm{x}$ & $\mathrm{x}$ \\
\hline Buscador múltiple & $\mathrm{x}$ & $\mathrm{x}$ & $\mathrm{x}$ & $\mathrm{x}$ & $\mathrm{x}$ & $\mathrm{x}$ & $\mathrm{x}$ & $\mathrm{x}$ & $\mathrm{x}$ & & $\mathrm{x}$ & $\mathrm{x}$ & $\mathrm{x}$ & $\mathrm{x}$ & $\mathrm{x}$ & $\mathrm{x}$ & $\mathrm{x}$ \\
\hline $\begin{array}{l}\text { Punto SPARQL/Compendio } \\
\text { datasets/API }\end{array}$ & $\mathrm{x}$ & $\mathrm{x}$ & & $\mathrm{x}$ & & $\mathrm{x}$ & $\mathrm{x}$ & $\mathrm{x}$ & & & $\mathrm{x}$ & $\mathrm{x}$ & & & & & $\mathrm{x}$ \\
\hline $\begin{array}{l}\text { Noticias / Información } \\
\text { adicional }\end{array}$ & $\mathrm{x}$ & $\mathrm{x}$ & & $\mathrm{x}$ & $\mathrm{x}$ & $\mathrm{x}$ & $\mathrm{x}$ & $\mathrm{x}$ & $\mathrm{x}$ & & $\mathrm{x}$ & $\mathrm{x}$ & $\mathrm{x}$ & $\mathrm{x}$ & $\mathrm{x}$ & $\mathrm{x}$ & $\mathrm{x}$ \\
\hline Estadísticas & $\mathrm{x}$ & & & & $\mathrm{x}$ & & & & & & & & & & & & $\mathrm{x}$ \\
\hline
\end{tabular}

NOTA: AND - Andalucía, ARA - Aragón, AST - Principado de Asturias, BAL - Islas Baleares, CAT - Cataluña, CNR - Canarias, CNT - Cantabria, CTL - Castilla y León, CTM - Castilla-La Mancha, EXT - Extremadura, GAL - Galicia, MAD - Comunidad de Madrid, MUR - Región de Murcia, NAV - Comunidad Foral de Navarra, RIO - La Rioja, VAL - Comunidad Valenciana, VAS - País Vasco.

cales, a las universidades y otras instituciones (que depositan $7.940,5.875,222$ y 33 conjuntos de datos respectivamente) según muestra data.gob.es.

Es necesario destacar, no obstante, un irregular desarrollo autonómico, ya que tres CC.AA., las que mayor incremento han experimentado en su catálogo durante el periodo 2013-2019, ofertan de manera conjunta dos terceras partes del total: País Vasco, 4.970 datasets; Aragón, 2.824 datasets; y Comunidad Foral de Navarra, 1.243 conjuntos de datos.

En cuanto a la tipología de los conjuntos de datos hemos observado que prima más la creación de valor que el fomento de la transparencia. Esto puede deberse a que los datos liberados por las distintas administraciones se dirigen más a estimular nuevas utilidades y servicios que a fomentar una forma innovadora de gobierno (Ferrer-Sapena y otros, 2011). Lamentamos la falta de estos datos públicos esenciales en las sociedades democráticas (Bertot y otros, 2010), que facilitarían la rendición de cuentas (Cerrillo-Martínez, 2014). El carecer de información reutilizable va a dificultar que la ciudadanía pueda monitorear e influir en los procesos gubernamentales (Meijer y otros, 2012), ya que aún queda mucho trabajo por hacer en España para que se cumpla la difusión de información transparente de forma satisfactoria (Beltrán-Orenes y Martínez-Pastor, 2017).

Varios estudios confirman la desatención a la transparencia activa de los portales de datos abiertos autonómicos. Curto-Rodríguez (2015) tan solo encuentra 40 conjuntos de datos (menos de un 1 $\%$ del total) asociados con un indicador de transparencia inspirado en la LTAIPyBG. García-García y Curto-Rodríguez (2018a) que valoran el desempeño autonómico ponderando la existencia de información por su facilidad reutilizadora y ponen de manifiesto que tan solo País Vasco roza el aprobado. Señalan, además, que las mejores puntuaciones de las CC.AA. presentan una asociación positiva con la competitividad, con un mayor gasto en educación y en sanidad, con régimen tributario foral o con el acceso a la autonomía mediante el mayor techo de 
competencias, y una relación negativa con una mayor fortaleza política del partido en el gobierno o el hecho de gobernar con mayoría absoluta.

Una nueva visita a los portales, coincidiendo con la entrada en vigor de la Ley para las Entidades Locales y Comunidades Autónomas en diciembre de 2015 (Curto-Rodríguez, 2017b), apunta a un incremento poco sustancial de los conjuntos de datos transparentes (hasta los 92 datasets), escaseando sistemáticamente ítems sensibles (Curto-Rodríguez, 2019a), y constatando que el destino de la información exigida por la LTAIPyBG fue los portales de transparencia y no los de datos abiertos (García-García y Curto-Rodríguez, 2019).

\subsection{Aplicaciones}

No tiene sentido disponer una enorme cantidad de documentos almacenados si no hay una política para hacerlos accesibles, fomentar su uso o promover la interacción con los ciudadanos (Bauhr y Grimes, 2014). Puesto que los datos abiertos son la infraestructura básica para la creación de negocios y de productos o servicios (Abella y otros, 2018), se deben valorar las aplicaciones que han sido elaboradas en base a la información disponible en los portales de open data autonómicos.

Tan solo hemos encontrado 92 apps que utilicen los datos suministrados por los portales de datos abiertos, lo que nos parece un escaso aprovechamiento de la iniciativa. Constatamos además que la mayor parte de las aplicaciones se desarrollan internamente por la propia CC.AA., aspecto inesperado, pero de similar resultado al informe de la reutilización de datos abiertos en España II (Abella y otros, 2019) que tras muestrear todos los portales de datos abiertos de España en el año 2019 concluyen que el 54,8 \% de los servicios digitales son elaborados por la propia administración. Y es que si la reutilización por parte de otros es la valía real de la información (Beltrán-Orenes y Martínez-Pastor, 2016), parece que la apuesta por la inteligencia colectiva y la innovación con valor público (Valenzuela Mendoza, 2013) no ocupa un lugar destacado desaprovechando las virtudes de la economía colaborativa según postulados como The Many Minds Principle" (Walsh y Pollock, 2007), "el human database" (Noveck, 2009) o el crowdsourcing (Surowiecki, 2005).

El estudio diferencia entre las apps que pretenden favorecer el día a día de las personas y las relacionadas con la rendición de cuentas. Los resultados muestran que tan solo un porcentaje de un $5 \%$ de las aplicaciones tienen como objetivo el fomento de la transparencia, hallazgos en línea con los de Curto-Rodríguez (2019b) y en evidente consonancia con el escaso número de datasets de- dicados a este cometido, como se ha manifestado en el anterior apartado.

\subsection{Interacción}

Las opciones de interacción guardan relación con la participación, el segundo pilar del gobierno abierto entendido y calificado como una propuesta políticamente correcta y de fomento democrático (Díaz Aldret, 2017). Se compone de un conjunto de variadas y nuevas formas de relación gobierno-ciudadanía posibilitadas gracias a las tecnologías de la información (Fuentes García y Serrano Doblás, 2019) y de las tecnologías sociales (Criado y Villodre, 2018).

Estas distintas opciones deberían conferir a los ciudadanos un mayor protagonismo comunicativo frente a los responsables de los portales de datos abiertos al poder hacer más sugerencias (Hernández-Pérez y García-Moreno, 2013). Curiosamente se trata de la única posibilidad disponible de forma mayoritaria, habilitada, generalmente, mediante el envío de un correo electrónico al coordinador de la iniciativa.

A pesar de que Criado Grande (2013) menciona que las redes sociales más utilizadas en las administraciones corresponden a aquellas más extendidas en la sociedad (Facebook, Twitter, Youtube, Flickr, Linkedin, etc.), tan solo hemos podido encontrar un Twiter específico para los datos abiertos, constatando que los perfiles de Facebook existentes en el periodo 2014-2018, según reflejaba Curto-Rodríguez (2019c), han sido eliminados. Señalamos, en último lugar, que tan solo siete CC.AA. ofrecen sindicación de contenidos vía feed RSS.

\subsection{Funcionalidades}

Esta categoría valora la existencia de un conjunto de características que favorecen un mejor aprovechamiento del portal de datos abiertos (diseño moderno y funcional, buscador múltiple punto SPARQL/compendio datasets/API, noticias / información adicional y estadísticas).

Podemos observar que varios aspectos están bien atendidos por las CC.AA. como el diseño moderno y funcional (salvo Baleares, que nos ha parecido poco operativo, y Principado de Asturias, con un diseño anticuado y un planteamiento deficiente), un buscador que favorezca localizar la información por distintos criterios (excepto Extremadura) o la puesta a disposición de información adicional (salvo Principado de Asturias y Extremadura). Las mayores diferencias las encontramos en la existencia de una consulta avanzada de los datasets (punto SPARQL/compendio datasets/API) solo presente en el $50 \%$ de las autonomías o en las estadísticas de 
Tabla V: Valoración global de los portales de datos abiertos autonómicos según orden de puntuación

\begin{tabular}{|l|c|c|c|c|c|}
\hline \multicolumn{1}{|c|}{ Comunidad Autónoma } & $\begin{array}{c}\text { Número de } \\
\text { datasets }\end{array}$ & $\begin{array}{c}\text { Aplicaciones } \\
\text { existentes }\end{array}$ & $\begin{array}{c}\text { Opciones de } \\
\text { interacción }\end{array}$ & Funcionalidad & $\begin{array}{c}\text { Puntuación } \\
\text { Total }\end{array}$ \\
\hline País Vasco & 4,00 & 0,38 & 1 & 1 & 6,38 \\
\hline Aragón & 2,27 & 1,63 & 1 & 0,8 & 5,70 \\
\hline Castilla y León & 0,39 & 2,38 & 2 & 0,8 & 5,57 \\
\hline Andalucía & 0,45 & 3,00 & 1 & 1 & 5,45 \\
\hline Cataluña & 0,47 & 2,00 & 1,5 & 0,8 & 4,77 \\
\hline Comunidad Foral de Navarra & 1,11 & 0,63 & 1 & 0,6 & 3,33 \\
\hline Galicia & 0,29 & 0,38 & 1 & 0,8 & 2,47 \\
\hline Comunidad de Madrid & 0,14 & 0,50 & 1 & 0,8 & 2,44 \\
\hline La Rioja & 0,26 & 0,50 & 0,5 & 0,6 & 1,86 \\
\hline Región de Murcia & 0,62 & 0,13 & 0,5 & 0,6 & 1,84 \\
\hline Cantabria & 0,29 & 0,00 & 0,5 & 0,8 & 1,59 \\
\hline Comunidad Valenciana & 0,40 & 0,00 & 0,5 & 0,6 & 1,50 \\
\hline Canarias & 0,15 & 0,00 & 0,5 & 0,8 & 1,45 \\
\hline Castilla-La Mancha & 0,22 & 0,00 & 0,5 & 0,6 & 1,32 \\
\hline Islas Baleares & 0,15 & 0,00 & 0,5 & 0,6 & 1,25 \\
\hline Principado de Asturias & 0,06 & 0,00 & 0,5 & 0,2 & 0,76 \\
\hline Extremadura & 0,02 & 0,00 & 0,5 & 0,2 & 0,72 \\
\hline
\end{tabular}

uso y reutilización del portal de datos (únicamente disponibles en Andalucía, Cataluña o País Vasco).

Una vez han sido analizadas las distintas categorías e ítems considerados por nuestro estudio, finalizamos este apartado procediendo a calcular la valoración global de los portales de datos abiertos de las CC.AA. españolas que podrían oscilar entre diez puntos de máxima y cero de mínima.

Dado que la función principal de los portales es el suministro de datasets, hemos destinado un $40 \%$ de la puntuación del indicador al número de conjunto de datos albergados de forma que la autonomía con más datasets recibirá 4 puntos y el resto una valoración proporcional al número de datasets respecto al líder. Al ser las aplicaciones la materialización más evidente de la utilidad de los datasets, le reservamos un $30 \%$ de la puntuación total (baremando de forma análoga al número de conjuntos de datos). Los otros dos apartados van a asignar puntuaciones iguales por cada subapartado que los componen, si bien, ya que la ponderación de las opciones de interacción será de un $20 \%$ y las funcionalidades de un $10 \%$, cada ítem atendido sumará 0,50 y 0,20 puntos, respectivamente. La valoración global se ofrece en la Tabla $V$ ofreciendo, para facilitar la visualización de los resultados, la figura 2 que señala de forma inequívoca las grandes diferencias existentes.
Figura 2: Valoración global de los portales de datos abiertos autonómicos por orden alfabético

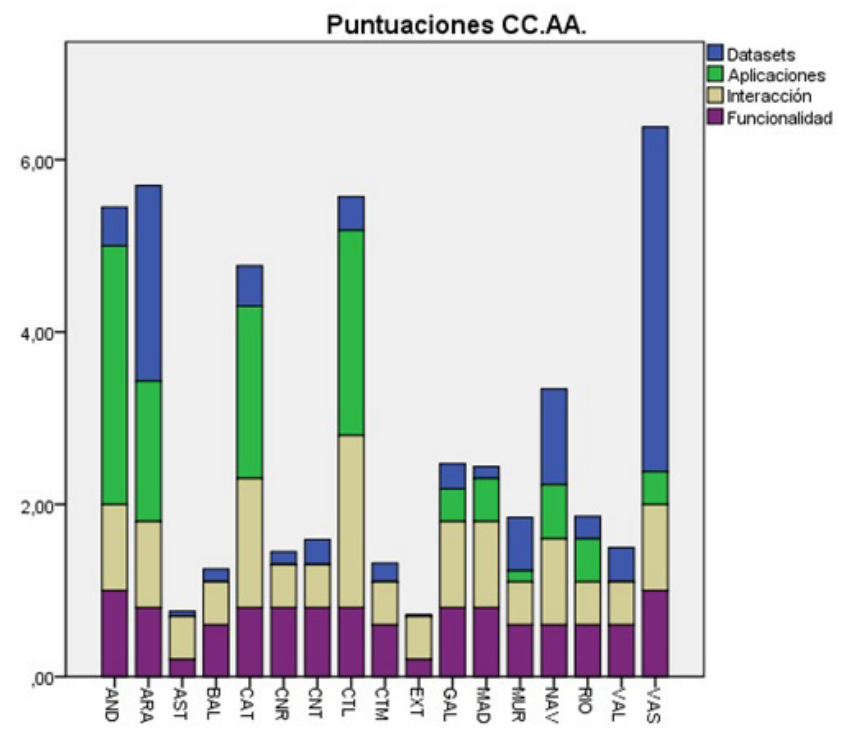

NOTA: AND - Andalucía, ARA - Aragón, AST - Principado de Asturias, BAL - Islas Baleares, CAT - Cataluña, CNR - Canarias, CNT - Cantabria, CTL - Castilla y León, CTM - Castilla-La Mancha, EXT - Extremadura, GAL - Galicia, MAD - Comunidad de Madrid, MUR - Región de Murcia, NAV Comunidad Foral de Navarra, RIO - La Rioja, VAL - Comunidad Valenciana, VAS - País Vasco. 


\section{CONCLUSIONES, LIMITACIONES Y FUTU- RAS LÍNEAS DE INVESTIGACIÓN}

Los portales de open data son el reflejo de una corriente internacional en favor de la apertura de datos gubernamentales a la que España no ha sido ajena, contando según datos.gob.es, con casi 300 iniciativas operativas. Al ser las CC.AA. el mayor suministrador nacional de datasets, hemos decidido estudiar, a finales de 2019, sus repositorios y valorarlos mediante cuatro apartados diferenciados.

El primer apartado son los datasets existentes, materia prima de la que se nutren distintos proyectos y servicios digitales. La media autonómica de los 14.000 conjuntos de datos encontrados sería de 823 datasets por autonomía, pero la distribución es terriblemente irregular ya que solo tres CC.AA. superan esa media: País Vasco, con casi 5.000 datasets; Aragón, cerca de los 3.000 datasets, y Comunidad Foral de Navarra, próxima a los 1.500 conjuntos de datos existiendo, en el extremo opuesto, autonomías como Extremadura, con tan solo 25 datasets, o Principado de Asturias con 72 .

Resulta difícil encontrar negocios donde la materia prima se ofrezca de forma gratuita, pero si aun así esta no se transforma en productos terminados listos para el consumo, el esfuerzo de las administraciones en su puesta a disposición de la información habrá sido estéril. Por ello, es necesario valorar, como segundo aspecto de nuestro estudio, las aplicaciones que han sido elaboradas con los conjuntos de datos de los portales autonómicos, encontrando 92 apps desarrolladas, lo que nos parece un desempeño deficiente, ya que un porcentaje inferior al $1 \%$ de los datasets se ven convertidos en aplicaciones. La media autonómica estaría en las 5,4 apps, pero de nuevo apreciamos una importante dispersión, ya que cuatro CC.AA. aportan el $80 \%$ de ellas: dos mediante exclusivo desarrollo interno (Andalucía, con 24 aplicaciones y Cataluña con 16 apps) y dos que se apoyan en el desarrollo externo impulsado por los concursos (Castilla y León y Aragón, con 19 y 13 aplicaciones, respectivamente). Es importante señalar que tan solo cinco de las 92 aplicaciones están relacionadas con el fomento de la transparencia (hallazgo en línea con los reducidos datasets asociados a la rendición de cuentas que se están divulgando).

La tercera sección contempla varias opciones que permiten interactuar con los responsables de los portales abiertos. Tan solo Castilla y León completa las cuatro opciones disponibles (oferta de contacto, blog o herramienta de votación de propuestas, Twitter y sindicación de contenidos vía RSS, o suscrip- ción de novedades), seguida por Cataluña con tres. En el extremo opuesto estarían nueve CC.AA. con tan solo un ítem completado, lo que pone de manifiesto la mayoritaria desatención de este apartado.

El cuarto aspecto, por el contrario, presenta una mejor valoración, siendo el diseño moderno y funcional de los portales de open data el ítem más atendido por las autonomías. Encontramos dos CC.AA. (País Vasco y Andalucía) que ofrecen las cinco utilidades buscadas; otras siete autonomías que ofrecen cuatro opciones; y seis CC.AA. que completan tres de los ítems. Tan solo Asturias y Extremadura ofrecen una única funcionalidad.

El ranking autonómico global elaborado pondera esos cuatro apartados de forma decreciente. Puesto que la principal función de los portales de open data es centralizar, clasificar, ordenar y hacer más accesible los conjuntos de datos allí albergados, el mayor porcentaje ( $40 \%$ ) se le asigna al número de datasets existentes. Un porcentaje del $30 \%$ se reserva a las aplicaciones elaboradas en base a esos conjuntos de datos, un $20 \%$ a las posibilidades de interacción habilitadas en el portal y el $10 \%$ restante a las diversas funcionalidades existentes.

Conseguimos, de esta forma, valorar los portales de datos abiertos de las autonomías españolas como en su día hicieron otros autores. En 2013 pudimos observar que "Open Data Euskadi es ejemplar y ha marcado el camino a otras como Cataluña, Navarra, Castilla y León o, más recientemente, Aragón, que quieren seguir sus pasos" (Gestores Públicos, 2013). Para la Fundación Orange y Arvo Consultores y Tecnología (2014), solo Aragón, Cataluña y País Vasco (de entre las 11 CC.AA. con portal operativo) eran merecedoras de recibir un galardón positivo a nivel global. Los líderes autonómicos eran, según el estudio de Vicente-Paños y Jordán-Alonso (2017), Aragón, Cataluña, Navarra y País Vasco.

Nuestra investigación llega a conclusiones muy parecidas a las anteriores, aunque emplee una métrica más completa, distintas ópticas y diferente horizonte temporal. Las mejores CC.AA. son País Vasco, Aragón, Castilla y León, Andalucía y Cataluña $(6,38$ - 5,70- 5,57- 5,45 - y 4,77 puntos, respectivamente). Les siguen, con una puntuación netamente inferior a los cinco puntos, la Comunidad Foral de Navarra, Galicia, Comunidad de Madrid $(3,33-2,47$ y 2,44$)$, y otras cuatro CC.AA. que reciben entre uno y dos puntos: Comunidad Valenciana, Canarias, Castilla-La Mancha, Islas Baleares $(1,50-1,45-1,32$ y 1,25 puntos, respectivamente). Cierran la clasificación Principado de Asturias y Extremadura que no llegan a alcanzar ni el $10 \%$ de la puntuación máxima otorgable $(0,76$ y 0,72 puntos). 
Es notorio que las puntuaciones son bajas y que la valoración global presenta, al igual que la desagregada por apartados, grandes diferencias autonómicas. País Vasco es líder en cuanto a datasets, Andalucía presenta un mayor número de aplicaciones, Castilla y León una mayor atención a las opciones de interacción y, Andalucía y País Vasco las que cuentan con un portal más funcional. Como vemos, no hay ninguna Comunidad Autónoma que destaque en todos los apartados, resultando curioso, que País Vasco, que ofrece más de un tercio de los conjuntos de datos, solo cuente con tres aplicaciones desarrolladas.

Es necesario señalar una doble limitación en el presente estudio. Por un lado, que se trata de una instantánea que quedará obsoleta (aunque nos habilite la posibilidad de realizar futuras valoraciones que permitan mostrar la evolución autonómica); y por otro, que el análisis descriptivo se basa en un indicador propio no exento de cierta subjetividad que pueda provocar que determinadas personas echen en falta algún apartado, que piensen que sobra alguno, o incluso que la ponderación aplicada es susceptible de mejora.

\section{REFERENCIAS}

Abella, A.; Ortiz-de-Urbina-Criado, M.; De-Pablos-Heredero, C. (2014). Meloda, a metric to assess open data reuse. El Profesional de la Información, 23(6), 582588. https://doi.org/10.3145/epi.2014.nov.04

Abella, A.: Ortiz-de-Urbina-Criado, M.: De-Pablos-Heredero, C. (2017). La reutilización de datos abiertos: una oportunidad para España. Disponible en: http://informecotec.es/media/INFORME REUTILIZACION-DE-DATOS.pdf [Fecha de consulta: 21/12/2019].

Abella, A.; Ortiz-de-Urbina-Criado, M.; De-Pablos-Heredero, C. (2018). Indicadores de calidad de datos abiertos: el caso del portal de datos abiertos de Barcelona. El Profesional de la Información, 27(2), 375-382. https://doi.org/10.3145/epi.2014.nov.04

Abella, A.; Ortiz-de-Urbina-Criado, M.; De-Pablos-Heredero, C.; Vidal Cabo, C.: Ferrer Sapena, A. (2019). La reutilización de datos en España II. Disponible en: https://www.desidedatum.com/informe-opendata/ [Fecha de consulta: 21/12/2019].

Alcaide Muñoz, L.; Rodríguez Bolívar, M. P.; Villamayor Arellano, C. L. (2019). Open Government Initiatives in Spanish Local Governments: An Examination of the State of the Art. En: Rodríguez Bolívar, M. P. Bwalya K. J.; Reddick C. G., (coords.), Governance Models for Creating Public Value in Open Data Initiatives pp. 123139. Springer, Cham. https://doi.org/10.1007/978-3030-14446-3_6

Aleixandre-Benavent, R.; Sapena, A. F.; Peset, F. (2019). Compartir los recursos útiles para la investigación: datos abiertos (open data). Educación Médica. https:// doi.org/10.1016/j.edumed.2019.07.004

Arquero Avilés, R.; Marco Cuenca, G. (2014). El Portal de datos abiertos de la Unión Europea: análisis y eva-
En todo caso, hemos pretendido elaborar una herramienta de carácter sintético, que cuantifique cuatro aspectos de los portales de datos abiertos autonómicos españoles, llenando un vacío en la literatura y habilitando múltiples líneas de investigación a seguir. Por ejemplo, valorar no solo el número de datasets, sino su facilidad reutilizadora (formatos y licencias en que se suministran), considerar, además de las aplicaciones existentes, el número de descargas o su grado de uso (lo que podría ser un proxy de su utilidad) o incorporar otras variables no cuantitativas (como la presencia de herramientas de marketing que favorezcan el empleo de la información y/o de los servicios digitales). De gran interés nos parecería abordar los determinantes que pudieran estar asociados a las diferencias observadas en las autonomías o desarrollar un análisis clúster con el cometido de identificar distintas tipologías subyacentes. La elaboración de una comparativa con otros países también sería muy necesaria, ya que permitirá valorar la situación real de España en cuanto a sus portales de datos abiertos. Estamos seguros que alguna de estas posibilidades se llevará a la práctica.

luación. Revista General de Información y Documentación, 24(1), 99-118.

Bastida, F.; Benito, B.; Guillamón, M. D.; Ríos, A. M. (2019). Tax mimicking in Spanish municipalities: expenditure spillovers, yardstick competition, or tax competition?. Public Sector Economics, 43(2), 115-139. http://www.pse-journal.hr/en/archive/ tax-mimicking-in-spanish-municipalities-expenditure-spillovers-yardstick-competition-or-tax-competition_4110/

Bauhr, M.; Grimes, M. (2014). Indignation or resignation: The implications of transparency for societal accountability. Governance, 27(2), 291-320. https://doi. org/10.1111/gove.12033

Beltrán-Orenes, P.; Martínez-Pastor, E. (2016). Grado de cumplimiento de las leyes de transparencia, acceso y buen gobierno y de reutilización de los datos de contratación de la administración central española. El Profesional de la Información, 25(4), 557-567. https:// doi.org/10.3145/epi.2016.jul.05

Beltrán-Orenes, P.; Martínez-Pastor, E. (2017). Organización de la información y sujetos obligados en el Portal de la Transparencia de España. El Profesional de la Información, 26(5), 983-994. https://doi.org/10.3145/ epi.2017.sep.19

Berners-Lee, T. (2010). Linked Data. World Wide Web Consortium. Disponible en: https://www.w3.org/2011/ gld/wiki/5 Star Linked Data [Fecha de consulta: 21/12/2019]

Bertot, J-C.; Jaeger, P. T.; Grimes, J. M. (2010). "Using ICTs to create a culture of transparency: Egovernment and social media as openness and anti-corruption tools for societies". Government Information Quar- 
terly, 27 (3), 264-271. https://doi.org/10.1016/j. giq.2010.03.001

Boix, A. (2015). "Transparencia, participación y procedimiento en la elaboración de disposiciones reglamentarias para un modelo de open government". En: Cotino-Hueso, Lorenzo; Sahuquillo-Orozco, José-Luis; Corredoira-Alfonso, Loreta (eds.). El paradigma del gobierno abierto. Retos y oportunidades de la participación, transparencia y colaboración, pp. 123-129. Madrid: Universidad Complutense de Madrid. https://gobiernoabiertovalencia.files.wordpress. com/2016/02/23-andres-boix_002.pdf [Fecha de consulta: 21/12/2019].

Cabo, D. (2013). Portales opendata como aeropuertos sin aviones: Disponible en: https://civio.es/novedades/2013/04/07/opendata/ [Fecha de consulta: 21/12/2019].

Cerrillo-Martínez, A. (2014). Los principios de los datos abiertos en la legislación española. IDP: Revista de Internet, Derecho y Política, (19), 62-77. https://doi. org/10.7238/idp.v0i19.2418

Cerrillo-Martínez, A. (2018). Datos masivos y datos abiertos para una gobernanza inteligente. El Profesional de la Información, 27(5), 1128-1135. https://doi. org/10.3145/epi.2018.sep.16

Clabo, N.; Ramos-Vielba, I. (2015). Reutilización de datos abiertos en la administración pública en España y uso de licencias-tipo. Revista Española de Documentación Científica, 38(3): e097. doi: http://dx.doi. org/10.3989/redc.2015.3.1206

Criado Grande, J. I. (2013). Redes sociales para unas administraciones y gobiernos abiertos. Desafíos para la innovación y la creación de valor público. Buen Gobierno 15 (1), 15-25. https://doi.org/10.35247/buengob_15_01

Criado Grande, J. I. (2016). Las administraciones públicas en la era del gobierno abierto. Gobernanza inteligente para un cambio de paradigma en la gestión pública. Revista de Estudios Políticos, (173), 245-275. https://doi.org/10.18042/cepc/rep.173.07

Criado, J. I.; Gil-Garcia, J. R. (2019). Creating public value through smart technologies and strategies. International Journal of Public Sector Management 32 (5), 438450. https://doi.org/10.1108/IJPSM-07-2019-0178

Criado, J. I.; Villodre, J. (2018). Comunicando datos masivos del sector público local en redes sociales. Análisis de sentimiento en Twitter. El Profesional de la Información, 27(3), 624-632. https://doi.org/10.3145/ epi.2018.may.14

Curto-Rodríguez, R. (2015). Los portales de datos abiertos autonómicos y la rendición de cuentas. Auditoría Pública: Revista de los Órganos Autónomos de Control Externo, 66, 75-83.

Curto-Rodríguez, R. (2017a). El impacto de la Ley 19/2013 de Transparencia, Acceso a la Información Pública y Buen Gobierno en la publicación de información relacionada con la rendición de cuentas en los portales de datos abiertos autonómicos españoles. Auditoría pública: revista de los Órganos Autónomos de Control Externo, 70, 143-152.

Curto-Rodríguez, R. (2017b). Los portales autonómicos de datos abiertos y la información relacionada con la rendición de cuentas: Punto de partida y situación tras la entrada en vigor de la ley 19/2013 de transparencia, acceso a la información pública y buen gobierno. Revista Española de la Transparencia, 5, 80-93.

Curto-Rodríguez, R. (2019a). ¿Qué información relacionada con la publicidad activa contienen los portales de datos abiertos autonómicos españoles? Un análisis detallado tras cuatro años de su eclosión. Revista Española de la Transparencia, 9, 177-198.

Curto-Rodríguez, R. (2019b). Reutilización de información pública y economía colaborativa. En Álvarez González, L. I.; Sanzo Pérez, M. J.; (dirs.). Actas del XI Congreso Internacional de Casos Docentes en Marketing Público y No Lucrativo. Oviedo. España: Universidad de Oviedo. [Fecha de consulta: 21/12/2019].

Curto-Rodríguez, R. (2019c). Opciones de interacción, participación y colaboración en los portales de datos abiertos autonómicos españoles para el período 2014-2018. Revista Buen Gobierno, 27.

Chatfield, A. T.; Reddick, C. G. (2017). A longitudinal cross-sector analysis of open data portal service capability: The case of Australian local governments. Government Information Quarterly, 34(2), 231-243. https://doi.org/10.1016/j.giq.2017.02.004

Díaz Aldret, A. (2017). Participación ciudadana en la gestión y en las políticas públicas. Gestión y Política Pública, 26(2), 341-379.

Federación Española de Municipios y Provincias (2017). Datos abiertos. Guía estratégica para su puesta en marcha. Conjuntos de datos mínimos a publicar (FEMP) y elaboración propia. Disponible en: http:// femp.femp.es/files/3580-1617-fichero/Gu\%C3\%ADa\%20Datos\%20Abiertos.pdf [Fecha de consulta: 21/12/2019].

Federación Española de Municipios y Provincias (2019). Datos abiertos FEMP 2019. 40 conjuntos de datos a publicar por las entidades locales. Disponible en: http://femp.femp.es/files/3580-1937-fichero/ DATOS\%20ABIERTOS\%20FEMP\%202019.pdf. [Fecha de consulta: 21/12/2019].

Ferrer-Sapena, A.; Peset, F.; Aleixandre-Benavent, R. (2011). Acceso a los datos públicos y su reutilización: open data y open government. El Profesional de la Información, 20(3), 260-269. https://doi.org/10.3145/ epi.2011.may.03

Ferrer-Sapena, A.; Sánchez-Pérez, E. A. (2013). "Open data, big data: ¿hacia dónde nos dirigimos?". Anuario ThinkEPI, 7, 150-156.

Fuentes García, R.; Serrano Doblás, J. A. (2019). El concejo abierto, posibilidad de mayor democracia y participación ciudadana. Gestión y Política Pública, 28(2), 441-472. https://doi.org/10.29265/gypp.v28i2.626

Fundación Orange, Arvo Consultores y Tecnología. (2014). Datos abiertos en las Comunidades Autónomas y sus mayores ayuntamientos 2014. Disponible en https://www.proyectosfundacionorange.es/docs/ eE2014/Datos Abiertos 2014.pdf. [Fecha de consulta: $21 / 12 / 2019]$

García-García, J.; Curto-Rodríguez, R. (2018a). Determinantes de la apertura de datos y rendición de cuentas en los gobiernos regionales españoles. Revista del CLAD Reforma y Democracia, 70, 163-198. 
García-García，J.; Curto-Rodríguez， R. (2018b). Divulgación de información pública de las Comunidades Autónomas españolas (2013-2017): Portal de datos abiertos, portal de transparencia y web institucional. El Profesional de la Información, 27(5), 10511060. https://doi.org/10.3145/epi.2018.sep.09

García-García, J.; Curto-Rodríguez, R. (2019). El ejercicio de la rendición de cuentas mediante portales de datos abiertos en las comunidades autónomas españolas. IDP: Revista de Internet, Derecho y Política, 29. https://www.raco.cat/index.php/IDP/article/view/373562

Gestores Públicos. (2013). Portales Open Data en las Comunidades Autónomas: Mucha propaganda pero todavía poca información útil. Disponible en: http:// gestores-publicos.blogspot.com/2013/04/open-data-mas-propaganda-que-informacion.html [Fecha de consulta: 21/12/2019]

Gobierno de España (2013). Ley de transparencia, acceso a la información pública y buen gobierno.

Gobierno de España (2019). Datos.gob.es, iniciativa de datos abiertos del gobierno de España. Disponible en: https://datos.gob.es/. [Fecha de consulta: 21/12/2019].

Gómez, N. D.; Méndez, E.; Hernández-Pérez, T. (2016). Social sciences and humanities research data and metadata: A perspective from thematic data repositories. El Profesional de la Información, 25(4), 545-555. https://doi.org/10.3145/epi.2016.jul.04

González-Limón, M.; Rodríguez-Ramos, A. (2019). Revisión del cumplimiento de los Datos Abiertos por los Ayuntamientos españoles en la iniciativa Aporta. Revista Española de Documentación Científica, 42 (4), e247. https://doi.org/10.3989/redc.2019.4.1605

Hernández-Pérez, T. (2016). En la era de la web de los datos: primero datos abiertos, después datos masivos. El Profesional de la Información, 25(4), 517-525. http://dx.doi.org/10.3145/epi.2016.jul.01

Hernández-Pérez, T.; García-Moreno, M. A. (2013). Datos abiertos y repositorios de datos: nuevo reto para los bibliotecarios. El Profesional de la Información, 22 (3), 259-263. http://dx.doi.org/10.3145/epi.2013. may. 10

Janowicz, K.; Hitzler, P.; Adams, B.; Kolas, D.; Vardeman, I. I. (2014). Five stars of linked data vocabulary use. Semantic Web, 5(3), 173-176. https://doi. org/10.3233/SW-140135

Kroes, N. (2012). From Crisis of Trust to Open Governing. Bratislava, 5 March 2012, SPEECH/12/149. Disponible en: https://ec.europa.eu/commission/ presscorner/detail/en/SPEECH_12 149. [Fecha de consulta: 21/12/2019].

Manfredi-Sánchez, J. L. (2017). Horizontes de la información pública. El profesional de la información, 26(3), 353-360. https://doi.org/10.3145/epi.2017. may. 01

Martínez-Méndez, F. J.; López-Carreño, R.; Pastor-Sánchez, J. A. (2014). Open data en las administraciones públicas españolas: categorías temáticas y apps. El Profesional de la Información, 23(4), 415-423. https://doi.org/10.3145/epi.2014.jul.10

Martínez Moya, D. (2015). Evaluación de los aspectos de usabilidad y reutilización de información en el
Portal de la Transparencia de España. Cuadernos de Gestión de Información, 5(1), 36-52. https://doi. org/10.3145/epi.2017.sep.19

Meijer, A. J.; Curtin, D.; Hillebrandt, M. (2012). Open government: connecting vision and voice. International Review of Administrative Sciences, 78(1), 10-29. https://doi.org/10.1177/0020852311429533

Melián González, A.; Apolinario Hidalgo, L.; Dorta Velázquez, J. A.; Quesada Díaz, M. L. (2018). Propuesta de metodología para el análisis de los recursos de las Comunidades Autónomas de régimen común: aplicación al periodo 2009-2017. Auditoría pública, 71, 115-124.

Mendo, C; Ramos, L. F.; Arquero, R.; Del Valle-Gastaminza, F.; Botezán, I.; Sánchez, R.; Tejada, C.; Peón, J. L.; Cobo, S.; Sala, A. (2013). Del acceso a la reutilización, del dato al documento: una visión conceptual de la información pública. Revista Española de Documentación Científica, 36(3):e013. doi: http://dx.doi. org/10.3989/redc.2013.3.957

Noveck, B. S. (2009). Wiki Government: How Technology Can Make Government Better, Democracy Stronger, and Citizens More Powerful. Washington D.C.: Brookings Institution Press.

Obama, B. (2009a): Memorandum for the Heads of Executive Departments and Agencies: Transparency and Open Government. Washington, DC. Disponible en: https://obamawhitehouse.archives.gov/the-pressoffice/transparency-and-open-government. [Fecha de consulta: 21/12/2019].

Obama, B. (2009b): Open Government Directive. Washington, DC. Disponible en: https://obamawhitehouse. archives.gov/open/documents/open-government-directive. [Fecha de consulta: 21/12/2019].

ONTSI- Observatorio Nacional de las Telecomunicaciones y de la Sociedad de la Información (2016). Caracterización del sector infomediario en España, Edición 2016. Disponible en: https://www.ontsi.red.es/ ontsi/sites/ontsi/files/Estudio\%20de\%20Caracterizaci\%C3\%B3n\%20del\%20Sector\%20Infomediario\%20 2016_0.pdf. [Fecha de consulta: 21/12/2019].

Open data inceptión (2019): oficial website. Disponible en https://opendatainception.io/. [Fecha de consulta: 21/12/2019].

Parks, W. (1957). Open Government Principle: Applying the Right to Know Under the Constitution. George Washington Law Review, 26 (1), 1-77.

Peset, F.; Aleixandre-Benavent, R.; Blasco-Gil, Y.; Ferrer-Sapena, A. (2017). Datos abiertos de investigación. Camino recorrido y cuestiones pendientes. Anales de documentación, 20(1). https://doi.org/10.6018/ analesdoc.20.1.272101

Ramos Simón, L. F.; Arquero Avilés, R.; Botezan, I.; Cobo Serrano, S.; Sala Sánchez Jiménez, A.; Jiménez, R. S.; Del Valle Gastaminza, F. (2012). De la reutilización de información del sector público a los portales de datos abiertos en Europa. BiD: Textos Universitaris de Biblioteconomia i Documentació, 29(3). https://doi. org/10.3989/redc.2011.3.825

Ramos-Simón, L. F. (2017). El uso de las licencias libres en los datos públicos abiertos. Revista Española de Documentación Científica, 40(3): e179. doi: http:// dx.doi.org/10.3989/redc.2017.3.1376 
Ruvalcaba-Gomez, E. A.; Criado J. I. : Sandoval-Almazan R.; Valle-Cruz, D. (2019). Understanding the Perspectives of Open Government: Exploratory Factor Analysis in Spain and Mexico. En actas del 20th Annual International Conference on Digital Government Research, pp. 507-508. Dubai, United Arab Emirates. https://doi. org/10.1145/3325112.3328216

Sandoval-Almazán, R.; Gil-Garcia, J. R. (2012). Are government internet portals evolving towards more interaction, participation, and collaboration? Revisiting the rhetoric of e-government among municipalities. Government Information Quarterly, 29, 72-81. https:// doi.org/10.1016/j.giq.2011.09.004

Sierra Rodríguez, J. (2018a). Una crítica al diseño de los organismos de garantía del derecho de acceso en las Comunidades Autónomas. Revista Jurídica de Castilla y León, 45, 73-112.

Sierra Rodríguez, J. (2018b). A modo de introducción: apuntes básicos sobre la ley de transparencia, acceso a la información pública y buen gobierno. En Sánchez de Diego Fernández de la Riva (Coord.): Apuntes sobre la transparencia. Madrid: Universidad Complutense. Disponible en: https://eprints.ucm.es/id/eprint/49600/1/ LIBRO\%202018\%20APUNTES\%20SOBRE\%20LA\%20 TRANSPARENCIA\%20\%282\%29.pdf [Fecha de consulta: 21/12/2019].

Subirats, J. (1995). Quince Años de Comunidades Autónomas en España. Luces y sombras de una realidad aún en discusión. Revista del CLAD. Reforma y Democracia, 4, 1-13.
Sunlight foundation. (2007). Eight principles of open government data. Disponible en: https://opengovdata. org/ [Fecha de consulta: 21/12/2019].

Surowiecki, J. (2005). The wisdom of crowds. Nueva York: Anchor Books.

Thorsby, J.; Stowers, G. N.L.; Wolslegel, K.; Tumbuan, E. (2017). Understanding the content and features of open data portals in American cities. Government Information Quarterly, 34(1), 53-61. https://doi.org/10.1016/j.giq.2016.07.001

Valenzuela Mendoza, R. E. (2013). La transparencia colaborativa una herramienta para un gobierno abierto. Buen Gobierno, 18(4), 65-82.

Vicente-Paños, A.; Jordán-Alfonso, A. (2017). Acceso a la información pública y su reutilización en las comunidades autónomas: evaluación de la reutilización de datos abiertos. El Profesional de la Información, 26(3), 381392. https://doi.org/10.3145/epi.2017.may.04

Vickery (2011) "Datos abiertos un motor para la innovación, el crecimiento y la gobernanza transparente". Comunicación de la Comisión al Parlamento Europeo, al Consejo, al Comité Económico y Social Europeo y al Comité de las Regiones. Disponible en: https://eur-lex.europa.eu/ legal-content/ES/TXT/PDF/?uri=CELEX:52011DC0882\&from $=$ ES. [Fecha de consulta: 21/12/2019].

Walsh, J. y Pollock, R. (2007). Open Data and Componentization. Presentación en XTech 2007. "The Ubiquitous Web", Paris, 15-18 mayo. Disponible en: http://assets. okfn.org/files/talks/xtech_2007/. [Fecha de consulta: 21/12/2019]. 